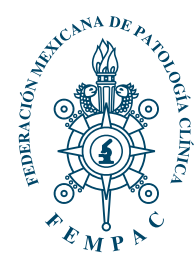

\title{
Distribución de los valores del Ct en la RT-PCR para SARS-CoV-2 al momento del diagnóstico en pacientes pediátricos mexicanos
}

\author{
Distribution of Ct values for SARS-CoV-2 RT-PCR at \\ of diagnosis in Mexican pediatric patients
}

Parra-Ortega Israel,* Carbajal-Franco Ebzadrel,* Vilchis-Ordoñez Armando,* Ángeles-Floriano Tania,* Nieto-Rivera Brenda,* López-Martínez Irma, ${ }^{*}$ Barrera-Badillo Gisela, ${ }^{\S}$ Klunder-Klunder Miguel,` López-Martínez Briceidall

Palabras clave: SARS-CoV-2, COVID-19, diagnóstico.

Keywords: SARS-CoV-2, COVID-19, diagnosis.

\footnotetext{
* Departamento de Laboratorio Clínico, Hospital Infantil de México Federico Gómez. México. ‡ Dirección de Diagnóstico y Referencia, Instituto de Diagnóstico y Referencia Epidemiológicos. México.

$\S$ Laboratorio de Virus Respiratorios, Instituto de Diagnóstico y Referencia Epidemiológicos. México.

^ Subdirección de Gestión de la Investigación, Hospital Infantil de México Federico Gómez. México.
}

\section{RESUMEN}

Introducción: La información generada por el laboratorio clínico para sustentar el diagnóstico de infección por el virus de SARS-CoV-2 basado en pruebas moleculares que apoyan el cuidado de los pacientes, el comportamiento de los resultados de las pruebas diagnósticas en pacientes pediátricos tiene algunas características importantes a destacar. Material y métodos: En el periodo de marzojulio de 2020 se procesaron 1,325 muestras de hisopados nasofaríngeos de pacientes pediátricos para realizar la identificación del virus SARS-CoV-2. En todos los casos se registraron los valores del ciclo de detección (cycle threshold por su ciclo en inglés) de los genes virales y el control interno. En este estudio la carga viral relativa se estimó sólo por el valor del Ct del ORF1ab. Resultados: Se identificaron $176(13.28 \%)$ resultados positivos, de los cuales 103/176 (58.5\%) fueron masculinos con edad de 0-17 años, con una media de seis años cinco meses y una mediana de cinco años tres meses, mientras que $73 / 176(41.5 \%)$ fueron del sexo femenino, con un intervalo de edad de 0-18 años, con una media de 6.2 años y una mediana de cuatro años cinco meses. En la distribución de los valores del $\mathrm{Ct}$ en los valores obtenidos por grupo de edad se observó una mediana de 31.9 a 34.01 y al comparar la distribución versus pacientes adultos no se observaron diferencias. Conclusión: Los valores del $\mathrm{Ct}$ al momento del diagnóstico identificados en niños mexicanos son mayores que lo reportado en otros sitios del mundo, lo que podría sugerir una menor cantidad de ARN viral en nuestra población al momento del diagnóstico.

\section{ABSTRACT}

Introduction: The information generated by the clinical laboratory to support the diagnosis of infection by the SARS-CoV-2 virus based on molecular tests, which supports the care of patients, the behavior of the results of diagnostic tests in pediatric patients has some characteristics important to highlight. Material and methods: From March to July of 2020, 1,325 nasopharyngeal swab samples from pediatric patients were processed to identify the SARS-CoV-2 virus. In all cases, the values of the cycle threshold $(\mathrm{Ct})$ of the viral genes and the internal control were recorded. In this study, the relative viral quantification was estimated only by the Ct value of ORF1ab gene. Results: 176 (13.28\%) positive results were identified, 103/176 (58.5\%) were male aged 0-17 years, with a mean of 6 years 5 months and a median of 5 years 3 months, while $73 / 176(41.5 \%)$ of the female sex, with an age range of 0-18 years with a mean of 6.2 years and a median of 4 years 5 months. The distribution of the $\mathrm{Ct}$ values. Values obtained by age group, a median of 31.9 to 34.01 was observed, and when comparing the distribution versus adult patients, no differences were observed. Conclusion: $\mathrm{Ct}$ values at the time of diagnosis, identified in Mexican children, are higher than those reported in other parts of the world, which could suggest a lower amount of viral RNA in our population at the time of diagnosis.

Citar como: Parra-Ortega I, Carbajal-Franco E, Vilchis-Ordoñez A, Ángeles-Floriano T, Nieto-Rivera B, López-Martínez I et al. Distribución de los valores del Ct en la RT-PCR para SARS-CoV-2 al momento del diagnóstico en pacientes pediátricos mexicanos. Rev Mex Patol Clin Med Lab. 2020; 67 (4): 176-182. https://dx.doi.org/10.35366/99463 
"l Subdirección de Servicios Auxiliares de Diagnóstico, Hospital Infantil de México Federico Gómez. México.

Correspondencia: Dra. Briceida LópezMartínez

Subdirección de

Servicios Auxiliares de

Diagnóstico,

Hospital Infantil

México Federico

Gómez.

Dr. Márquez Núm.

162, Col. Doctores,

06720,

Alcaldía Cuauhtémoc, CDMX, México. Tel: 5228-9917, ext. 2260

E-mail: blopez@

himfg.edu.mx

Recibido:

28/01/2021

Aceptado:

24/02/2021

\section{INTRODUCCIÓN}

$\neg$ n n el mes de diciembre de 2019 se presentó una serie de casos de neumonía atípica en la ciudad de Wuhan, provincia de Hubei, China. Estos casos fueron causados por un nuevo $\beta$-coronavirus denominado coronavirus del síndrome respiratorio agudo severo (SARS) 2 (SARS-CoV-2) por el Comité Internacional en Taxonomía de Virus (ICTV). ${ }^{1-4}$ Los coronavirus (CoVs) son virus envueltos cuyo genoma está constituido de una sola cadena de ácido ribonucleico (ARN) de polaridad positiva, cuatro géneros constituyen la familia Coronaviridae: los alfa-coronavirus, beta-coronavirus, gamma-coronavirus y delta-coronavirus. ${ }^{5}$ Los CoVs se distribuyen ampliamente en los seres humanos y otros mamíferos ocasionando enfermedades de afectación variable, desde una gripe común hasta neumonía. Sin embargo, en algunos individuos provocan un síndrome respiratorio grave, entre 10 y $40 \%$ de los pacientes requieren de ventilación mecánica ocasionando la muerte en los casos más severos. $^{3-8}$ Los datos actuales indican que los niños parecen ser el grupo etario menos afectado y la enfermedad es menos severa. Los niños, sin embargo, pueden cursar una infección asintomática y también una prolongada diseminación viral fecal, lo que puede contribuir a la diseminación de la pandemia. ${ }^{6}$

Dentro de las estrategias descritas por la Organización Mundial de la Salud (OMS) y la Secretaría de Salud en nuestro país para el diagnóstico y atención destacan la implementación de sistemas para el diagnóstico de los casos, la identificación oportuna de casos sospechosos, la toma y el envío de muestras a laboratorios autorizados, y la implementación de protocolos de detección molecular para SARS-CoV-2 de acuerdo con la capacidad de cada laboratorio. Por ello, la información que genera el laboratorio clínico para el apoyo en el diagnóstico basado en pruebas moleculares es de suma importancia para la atención oportuna de los pacientes, así como para la toma de decisiones en el manejo epidemiológico de esta pandemia. ${ }^{9-12}$ En nuestro caso, nos enfocaremos en la población pediátrica, la cual puede representar entre 1 y $5 \%$ de los casos según informes de otros países. ${ }^{3-9}$ A menudo estos pacientes cursan con un cuadro clínico menos grave en comparación con los adultos y las muertes en este tipo de pacientes han sido extremadamente raras. ${ }^{3-9}$

El objetivo del presente trabajo es describir la información recopilada dentro del proceso analítico de la prueba molecular utilizada en la identificación de SARS-CoV-2 en el laboratorio clínico del Hospital Infantil de México Federico Gómez, con base en los protocolos de diagnóstico molecular establecidos y recomendados por el Instituto de Diagnóstico y Referencia Epidemiológicos (InDRE).

\section{MATERIAL Y MÉTODOS}

En el periodo comprendido de marzo-julio de 2020 se incluyeron las muestras de todos los pacientes pediátricos con síntomas de infección por COVID-19 del Hospital Infantil de México Federico Gómez. Los hisopados (nasofaríngeo y orofaríngeo) se colectaron durante la primera semana del inicio de los síntomas, los cuales fueron recolectados utilizando hisopos de fibra sintética (Dracón flocados o poliéster). Las muestras se colocaron en $2.0 \mathrm{~mL}$ de solución salina balanceada de Hanks (HBSS) 1X (Gibco) y se mantuvieron a $4{ }^{\circ} \mathrm{C}$ durante un periodo no mayor de 24 horas hasta su procesamiento. El ARN total fue extraído y purificado empleando el robot QIAcube (QIAGEN) y el QIAamp ${ }^{\circledR}$ Viral RNA mini kit (QIAGEN) siguiendo los protocolos del fabricante. La detección de los genes virales ORF1ab, nucleocápside (NC), espícula (S) y del gen humano ARNasa P (control interno) se realizó mediante RT-PCR en tiempo real con los kits Detection kit for 2019 Novel Coronavirus (2019-nCoV) RNA (PCR-Fluorescence Probing) (Da An Gene Company Limited of Sun Yat-Sen University) y TaqMan 2019-nCoV Assay Kit v1 (Thermo Fisher Scientific). Los termocicladores QuantStudio 5 System (ABI/Life Technologies) equipado con el QuantStudio Design \& Analysis Software versión 2.3.3 y el CFX96 real-time system (BIO-RAD) se utilizaron para la amplificación y detección del material genético siguiendo las recomendaciones de los fabricantes y los lineamientos operativos vigentes establecidos por el InDRE. ${ }^{9}$ En todos los casos se registraron los valores del cycle threshold (Ct) de los genes virales y el control 
interno. En este estudio la carga viral relativa se estimó sólo por el valor del Ct del ORF1ab (Tabla 1 y Figura 1).

En los casos positivos a la presencia del virus SARSCoV-2 se realizó el análisis de la distribución de la carga viral relativa dentro de los diferentes grupos de edad en el grupo de pacientes pediátricos. Con el objetivo de comparar la distribución del valor del Ct (utilizado para estimar la cantidad de ARN viral) obtenido en pacientes menores de 18 años versus los pacientes adultos se incluyeron las características de 122 pacientes mayores de 18 años identificados como positivos a la presencia del virus SARS-CoV-2 en el mismo periodo de tiempo.

Se realizó un análisis estadístico descriptivo con los datos obtenidos a partir de los ensayos de laboratorio, utilizando el sistema informático GraphPad Prism 6.0 para Windows.

\section{RESULTADOS}

Se realizó la búsqueda del virus SARS-CoV-2 en 1,325 pacientes pediátricos (edad de 0 a 18 años, con una media de edad de 6.9 años), la población pediátrica que se atiende en nuestro instituto es la que recibe atención de tercer nivel. Dentro de las características de estos pacientes, $66 \%$ se atendieron por consulta externa, $77 \%$ de los hospitalizados coexistieron con comorbilidades, los grupos de enfermedades más frecuentes son las neoplasias, enfermedades cardiovasculares y enfermedades pulmonares.

Fueron positivos para la prueba 176 (13.3\%) pacientes, de los cuales 103/176 (58.5\%) fueron del sexo masculino. De todos los pacientes positivos se estimó la distribución de carga viral relativa de acuerdo con el género y la edad, así como el momento en que se realizó la detección (mes del diagnóstico). De acuerdo al valor del Ct (utilizado para estimar la cantidad de ARN viral) se observó que 13.1\% de los pacientes positivos presentaron carga viral muy alta, carga viral alta $9.1 \%$, carga viral media $14.2 \%$ y carga viral

\begin{tabular}{|c|c|c|}
\hline \multicolumn{3}{|c|}{$\begin{array}{l}\text { Tabla 1: Distribución de la cantidad de ARN } \\
\text { viral al momento del diagnóstico (carga viral } \\
\text { relativa) en } 176 \text { pacientes diagnosticados en el } \\
\text { Hospital Infantil de México Federico Gómez. }\end{array}$} \\
\hline $\begin{array}{l}\text { Valor del Ct (utilizado para estimar } \\
\text { la cantidad de ARN viral) }\end{array}$ & $n$ & $\%$ \\
\hline Muy alta $(\leq 20)$ & 23 & 13.1 \\
\hline Alta (20-24.9) & 16 & 9.1 \\
\hline Media (25-29.9) & 25 & 14.2 \\
\hline Baja (> 30) & 112 & 63.6 \\
\hline
\end{tabular}

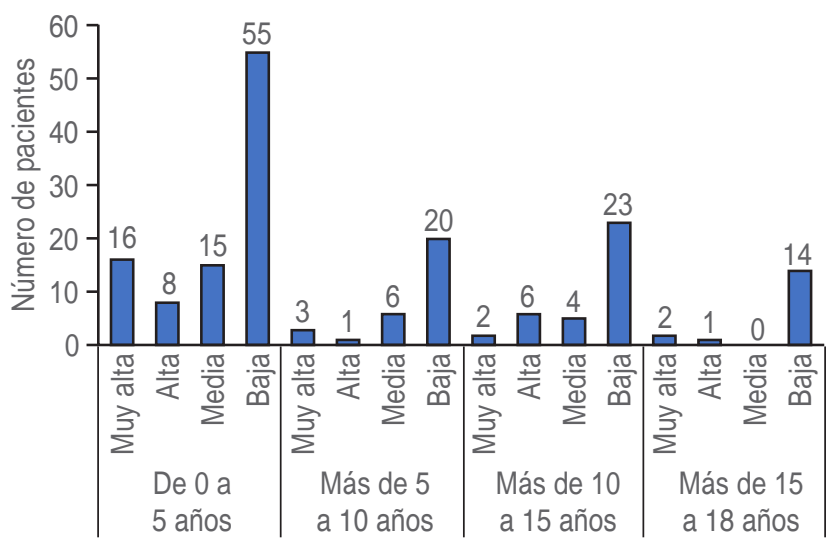

Figura 1: Distribución de la cantidad de ARN viral al momento del diagnóstico (carga viral relativa) de acuerdo con la edad en 176 pacientes diagnosticados en el Hospital Infantil de México Federico Gómez.

baja $63.6 \%$ (Tabla 1). La distribución de acuerdo al valor del Ct (utilizado para estimar la cantidad de ARN viral) por grupo de edad se muestra en la Figura 1.

Además, el valor de la carga viral relativa de acuerdo con los Ct no presentó diferencias por sexo (Figura 2A). Al comparar la distribución de los valores del Ct de los pacientes pediátricos por grupo de edad no se observaron diferencias, adicionalmente se compararon a los 176 pacientes pediátricos positivos a SARS-CoV-2 versus 122 resultados positivos de pacientes mayores de 18 años identificados en el mismo periodo (abril-julio de 2020), y tampoco se observaron diferencias estadísticamente significativas (Figura 2B).

Dentro de los pacientes pediátricos positivos, el valor del Ct presentó una diferencia en la distribución entre los meses de abril y julio (pruebas de Kruskal-Wallis $p<$ 0.0005), en el mes de mayo se evidenció que al diagnóstico la cantidad de ARN fue mayor comparada con otros meses (comparación múltiple, $\mathrm{p}=0.0008$ ); el aumento en el Ct muestra un aumento progresivo, lo cual significa que la cantidad de ARN viral al momento del diagnóstico fue disminuyendo gradualmente (Figura 3).

\section{DISCUSIÓN}

Los resultados observados en nuestro grupo de pacientes difieren en varios aspectos a lo reportado por HealdSargent y colaboradores, ${ }^{13}$ donde el valor de la mediana del Ct reportado es de 11.1 y de 6.5 en la población menor de cinco años, mientras que en nuestro estudio se observó un valor de la mediana de 32.5 y en la distribución de los valores obtenidos por grupo de edad se observó un valor de la mediana que va de 31.9 a 34.01 
de acuerdo al grupo de edad, por lo que inferimos que en todos los pacientes pediátricos positivos a la presencia del virus SARS-CoV-2, la cantidad de ARN viral fue baja. Ante esta situación se hace imperativo conocer los mecanismos fisiopatológicos que ocurren en la población pediátrica, ya que al no conocer exactamente lo que ocurre en ellos no podemos sacar conclusiones de lo que está ocurriendo en los pacientes identificados versus otros estudios publicados. ${ }^{13,14}$

Es necesario considerar que dentro del proceso analítico existen muchas variables que pueden modificar circunstancialmente los resultados en los Ct. Si bien el resultado final es cualitativo (positivo o negativo), existe la posibilidad de que las diferentes publicaciones que se encuentran en la comunidad científica no sean comparables por las siguientes condiciones o características: 1 ) tipo de material usado en la toma de muestra (hisopos y viales de transporte), 2) los días a partir del inicio de los síntomas en que se tomó la muestra (en nuestro caso fue durante la primera semana del inicio de los síntomas), 3) la metodología utilizada en la extracción de ARN y el rendimiento obtenido, 4) los protocolos de detección y los genes virales incluidos (nosotros realizamos la detección de los genes virales ORF1ab, NC, S). Por lo anterior, es necesario implementar procesos de control de calidad interlaboratorio y reducir al mínimo la variabilidad analítica y en dado caso llegar a tener pruebas cuantitativas de ARN viral.

Otra posible explicación a estos resultados es que los pacientes pediátricos mexicanos, por razones genéticas o biológicas, podrían presentar diferencias en la expresión

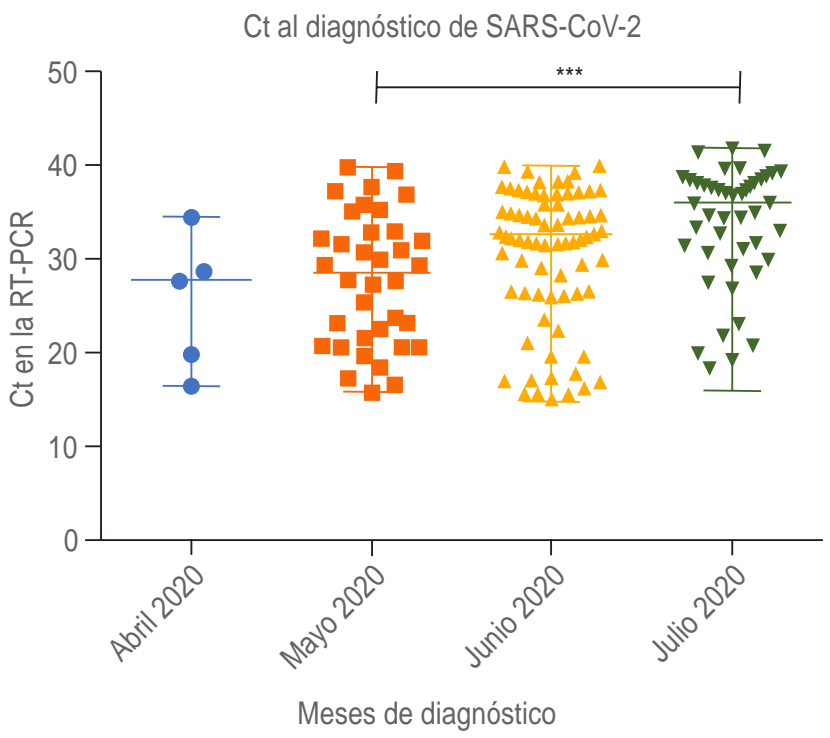

Figura 3: Descripción del Ct en la detección de SARS-CoV-2 en pacientes pediátricos.

del receptor celular asociado con los mecanismos de infección viral. De manera interesante, se ha demostrado que la expresión del receptor de la enzima convertidora de angiotensina II (ACE2), que es el receptor celular del SARS-CoV-2 en el epitelio nasal, depende de la edad del individuo, por lo tanto los niños expresan menos ACE2 cuando son comparados con la población adulta. Esta afirmación descrita por Bunyavanich $S$ y colaboradores ${ }^{14}$ ha generado diversas hipótesis sobre la participación de
Figura 2:

Distribución de la cantidad de ARN viral al momento del diagnóstico (carga viral relativa), $\mathbf{A})$ de acuerdo con el género y $\mathbf{B}$ ) en los diferentes grupos de edad.
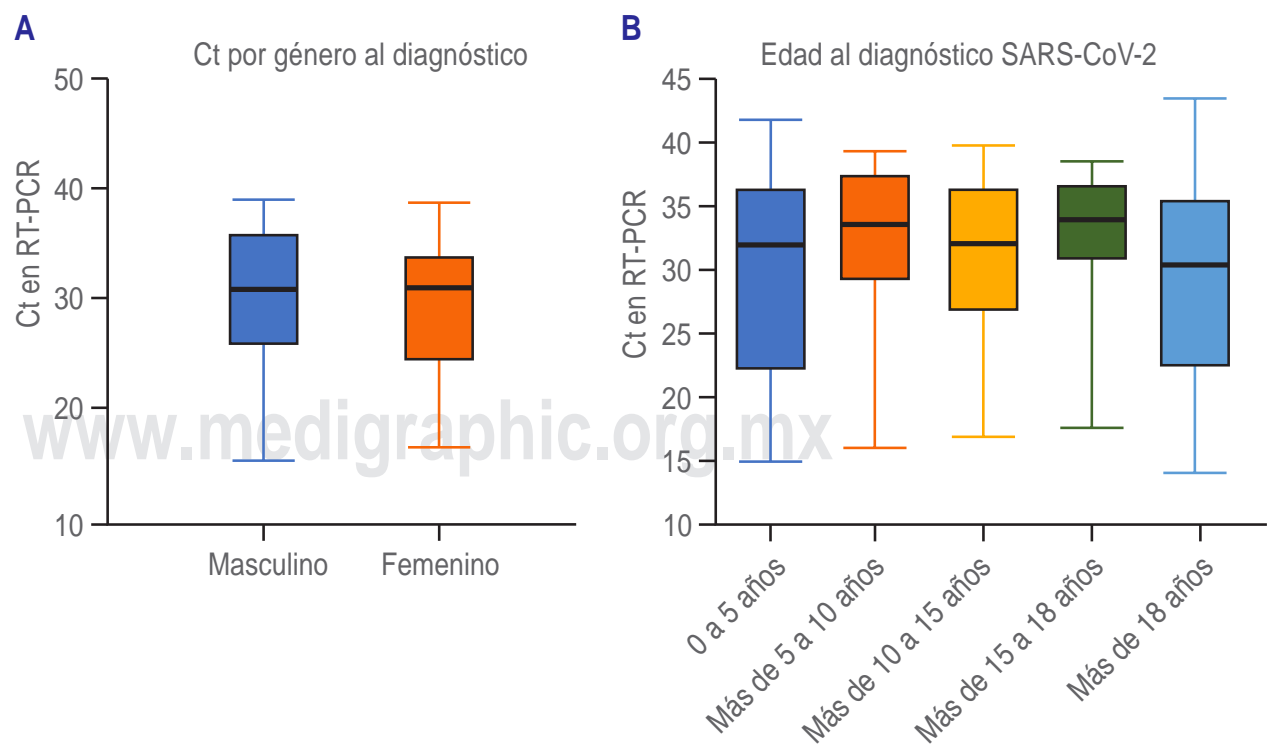
ACE2 en la gravedad de la enfermedad ${ }^{15-19}$ y creemos que esto condiciona a que los pacientes pediátricos presenten una baja cantidad de ARN viral al diagnóstico. Además, se ha encontrado que la expresión de ACE2 en las células pulmonares es diferente entre los grupos étnicos, siendo mayor en la población asiática comparada con la población de raza caucásica y afroamericana como lo describe Vinciguerra $M ;{ }^{20}$ sin embargo, no existe información disponible de la población latina.

Con respecto a las variaciones genéticas que pueden llegar a tener los virus, y en el caso especial del virus SARS-CoV-2, no contamos con evidencias que sustenten en estos momentos que las diferentes cepas circulantes en nuestro país tengan alguna particularidad en su comportamiento y por ende la patogénesis de la enfermedad. Lo que se ha reportado ${ }^{21}$ es el análisis genómico de las primeras variantes del SARS-CoV-2 introducidas en México, se ha realizado un análisis que demostró que el primer caso identificado en México pertenece al subclase A2a (linaje G) y está estrechamente relacionado con los aislados de Finlandia, Alemania y Brasil. Otro dato importante es que los primeros casos identificados de pacientes tienen antecedentes de viajes a Italia.

También como parte de la vigilancia epidemiológica y molecular que se realiza en nuestro país, se ha publicado la secuencia completa del genoma de 17 aislado de SARS-CoV-2 correspondiente a los primeros casos muestreados en México. La filogenómica global y local, junto con análisis mutacionales, reveló de manera consistente que estas secuencias virales se distribuyen dentro de dos linajes conocidos, el linaje A/G del SARS-CoV-2, que contiene principalmente secuencias de América del Norte, y el linaje $55 \mathrm{~B} / \mathrm{S}$ que contiene en esencia secuencias de Europa. Dentro de este grupo mexicano también se identificó un cambio de aminoácido H49Y en la proteína pico. Esta mutación es una homoplasia que se produce de forma independiente a través del tiempo y el espacio, y puede funcionar como un marcador molecular para seguir cualquier propagación adicional de estas variantes virales por todo el país. ${ }^{21,22}$

También debemos considerar que existe la posibilidad de que este fenómeno sea consecuencia de las diferentes estrategias diagnósticas implementadas en el país y específicamente en las áreas clínicas de nuestro instituto, pues se están identificando pacientes en etapas tempranas. Al observar el comportamiento del Ct obtenido en los pacientes y descrito en la Figura $2 B$, podríamos pensar que la cantidad de virus circulante es cada día menor; sin embargo, de esta especulación aún no existen datos que afirmen o rechacen el planteamiento, es por ello que dejamos esta pregunta o cuestionamiento sin respuesta alguna.
Ahora bien, se debe considerar detenidamente lo descrito en una serie de publicacione ${ }^{23-25}$ que refieren de manera general que los pacientes positivos al virus SARSCoV-2 por biología molecular presentan una carga viral de $10^{4}$ a $10^{8}$ copias/mililitro (que se estima cuantificando la presencia de un gen viral) en hisopados nasofaríngeos y saliva al momento del diagnóstico y que en los pacientes con enfermedad leve el valor máximo de la carga viral se puede detectar entre los cinco o seis días de iniciados lo síntomas, después disminuye hasta desaparecer al día 10 aproximadamente. Por otro lado existen algunos pacientes en quienes puede detectarse el virus después del día 10 de iniciados los síntomas y la carga viral identificada puede ser de 100 a 1,000 veces menor que la detectada en la prueba inicial diagnóstico. La estimación de la carga viral en estos momentos no se realiza en nuestro hospital con la cuantificación absoluta del virus, sólo se reporta o informa una carga viral relativa, la cual se basa en los $\mathrm{Ct}$ que se reportan en los análisis de los genes identificados según el protocolo de detección.

Nosotros proponemos que estos hallazgos tienen un fondo inmunológico por lo siguiente:

1. La inmunidad innata sirve como la primera línea de defensa antiviral y es esencial para la inmunidad a los virus. Actualmente no se tiene una certeza en los diferentes mecanismos de participación de la inmunidad innata frente a SARS-CoV-2. ${ }^{26}$ Sin embargo, basados en los modelos tradicionales de la inmunología y las interacciones descritas entre los virus y sus hospederos en los diferentes coronavirus es probable que el SARS-CoV-2 recapitule muchos de los mecanismos que involucran otros coronavirus, éstos basados en la homología de secuencia compartida. Dentro de los mecanismos conservados de inmunidad innata como son los receptores de reconocimiento de patrones (PRR), los receptores tipo Toll (TLR) y posterior a la activación de PRR, se genera una señalización que desencadenan la secreción de citocinas e interferones, siendo las tipo I y III las más importantes en la inmunidad contra el virus, junto con la liberación del factor de necrosis alfa (TNF- $\alpha$ ) e interleucinas 1, 6 y 18 , que conjuntamente con los mecanismos efectores de las células natural killer (NK) inducen programas de eliminación de células infectadas y la potencialización del sistema inmunológico adaptativo. De manera hipotética consideramos que estos mecanismos descritos influyeron en el valor del Ct obtenido y por ende, estimamos que se trata de una cantidad de ARN viral al momento del diagnóstico. ${ }^{26-28}$ 
Otra parte importante es la participación de las células T en la contención y eliminación del virus, recientemente se ha descrito que los componentes celulares de la inmunidad tienen un papel importante en el mecanismo de defensa ante el virus SARS-CoV-2 y por ende, en la presentación clínica de la enfermedad. Ya se han identificado células T específicas de SARSCoV-2 en humanos, ${ }^{29,30}$ pero aún no se ha esclarecido del todo su participación en la defensa contra el virus SARS-CoV-2, no se ha puesto de manifiesto hasta qué punto las características de la respuesta inmunitaria de las células T están asociadas con el curso clínico de COVID-19. Un estudio realizado en Estocolmo, Suecia describe que en la fase aguda de la enfermedad hay participación de células T específicas del SARS-CoV-2 y que las células participantes mostraron un fenotipo citotóxico que se correlacionó con varios marcadores clínicos usados para medir la gravedad de la enfermedad y además, en la etapa de convalecencia las células T específicas de SARS-CoV-2 eran polifuncionales, lo que demuestra que el SARS-CoV-2 provoca células T de memoria altamente funcionales, destacando la importancia de estos fenotipos de células $T$ en los individuos con antecedentes de COVID-19 leve y asintomático. Por lo anterior, consideramos que esta actividad de las células T puede, de manera conjunta con los mecanismos de la inmunidad innata, contener la multiplicación viral, por lo que en nuestros pacientes estudiados observamos una cantidad de ARN viral al momento del diagnóstico. ${ }^{27-31}$

Hoy en día que se ha comenzado a estudiar el genoma del virus, se empiezan a generar publicaciones sobre las cepas circulantes y sus características. ${ }^{21,22}$

Los valores del Ct al momento del diagnóstico identificados en niños mexicanos son mayores que los reportados en otros sitios del mundo, lo que podría sugerir una menor cantidad de ARN viral en nuestra población al momento del diagnóstico.

Fuente de financiamiento: Este proyecto fue parcialmente financiado por CONACYT-PAACTI/312936.

\section{Conflicto de interés: Ninguno.}

\section{REFERENCIAS}

1. Xu Z, Shi L, Wang Y, Zhang J, Huang L, Zhang C et al. Pathological findings of COVID-19 associated with acute respiratory distress syndrome. Lancet Respir Med. 2020; 8 (4): 420-422. doi: 10.1016/ S2213-2600(20)30076-X.

2. Colaneri M, Sacchi P, Zuccaro V, Biscarini S, Sachs M, Roda S et al. Clinical characteristics of coronavirus disease (COVID-19) early findings from a teaching hospital in Pavia, North Italy, 21 to 28 February 2020. Euro Surveill. 2020; 25 (16): 2000460.
3. Helmy YA, Fawzy M, Elaswad A, Sobieh A, Kenney SP, Shehata AA. The COVID-19 pandemic: a comprehensive review of taxonomy, genetics, epidemiology, diagnosis, treatment, and control. J Clin Med. 2020; 9 (4): 1225.

4. Huang C, Wang Y, Li X, Ren L, Zhao J, Hu Y et al. Clinical features of patients infected with 2019 novel coronavirus in Wuhan, China. Lancet. 2020; 395 (10223): 497-506.

5. Hilgenfeld R, Peiris M. From SARS to MERS: 10 years of research on highly pathogenic human coronaviruses. Antiviral Res. 2013; 100 (1): 286-295. doi: 10.1016/j.antiviral.2013.08.015.

6. Cai J, Sun W, Huang J, Gamber M, Wu J, He G. Indirect virus transmission in cluster of COVID-19 cases, Wenzhou, China, 2020. Emerg Infect Dis. 2020; 26 (6): 1343-1345. doi: 10.3201/ eid2606.200412.

7. Mustafa NM, A Selim L. Characterisation of COVID-19 pandemic in paediatric age group: a systematic review and meta-analysis. J Clin Virol. 2020; 128: 104395. doi: 10.1016/j.jcv.2020.104395.

8. Rodriguez-Morales AJ, Cardona-Ospina JA, Gutiérrez-Ocampo E, Villamizar-Peña R, Holguin-Rivera Y, Escalera-Antezana JP et al. Clinical, laboratory and imaging features of COVID-19: A systematic review and meta-analysis. Travel Med Infect Dis. 2020; 34: 101623.

9. Corman VM, Landt O, Kaiser M, Molenkamp R, Meijer A, Chu DK et al. Detection of 2019 novel coronavirus (2019-nCoV) by real-time RT-PCR. Euro Surveill. 2020; 25 (3): 2000045. doi: 10.2807/15607917.ES.2020.25.3.2000045.

10. World Health Organization. Laboratory testing for coronavirus disease 2019 (COVID-19) in suspected human cases. 2020. [Fecha de acceso 27 de 04 de 2020] Available in: https://apps.who.int/iris/ rest/bitstreams/1271387/retrieve.

11. Parra Ortega I, Vilchis Ordoñez A, López Martínez B, Ángeles Floriano A. Recomendaciones analíticas para la Identificación de SARS-CoV-2 por RT-PCR en pacientes pediátricos. Bol Med Hosp Inf. 2020 [en prensa].

12. Ai T, Yang Z, Hou H, Zhan C, Chen C, Lv W et al. Correlation of Chest CT and RT-PCR testing for coronavirus disease 2019 (COVID-19) in China: a report of 1014 cases. Radiology. 2020; 296 (2): E32-E40. doi: 10.1148/radiol.2020200642.

13. Heald-Sargent T, Muller WJ, Zheng X, Rippe J, Patel AB, Kociolek LK. Age-related differences in nasopharyngeal severe acute respiratory syndrome coronavirus 2 (SARS-CoV-2) levels in patients with mild to moderate coronavirus disease 2019 (COVID-19). JAMA Pediatr. 2020; 174 (9): 902-903. doi: 10.1001/ jamapediatrics.2020.3651.

14. Bunyavanich S, Do A, Vicencio A. Nasal gene expression of angiotensin-converting enzyme 2 in children and adults. JAMA. 2020; 323 (23): 2427-2429.

15. Patel AB, Verma A. Nasal ACE2 levels and COVID-19 in children. JAMA. 2020; 323 (23): 2386-2387. doi: 10.1001/jama.2020.8946.

16. Study to determine incidence of novel coronavirus infection in US children begins. National Institutes of Health website. Published May 4, 2020. Accessed Sep 26, 2020. Available in: https://www. nih.gov/news-events/news-releases/studydetermine-incidencenovel-coronavirus-infectionus-children-begins

17. Hoffmann $M$, Kleine-Weber $\mathrm{H}$, Schroeder $\mathrm{S}$, Krüger $\mathrm{N}$, Herrler T, Erichsen S et al. SARS-CoV-2 cell entry depends on ACE2 and TMPRSS2 and is blocked by a clinically proven protease inhibitor. Cell. 2020; 181 (2): 271-280.e8. doi: 10.1016/j.cell.2020.02.052.

18. Jackson DJ, Busse WW, Bacharier LB, Kattan M, O'Connor GT, Wood RA et al. Association of respiratory allergy, asthma, and expression of the SARS-CoV-2 receptor ACE2. J Allergy Clin Immunol. 2020; 146 (1): 203-206.e3. doi: 10.1016/j.jaci.2020.04.009.

19. Camiolo M, Gauthier M, Kaminski N, Ray A, Wenzel SE. Expression of SARS-CoV-2 receptor ACE2 and coincident host 
response signature varies by asthma inflammatory phenotype. J Allergy Clin Immunol. 2020; 146 (2): 315-324.e7. doi: 10.1016/j. jaci.2020.05.051.

20. Vinciguerra M, Greco E. SARS-CoV-2 and black population: ACE2 as shield or blade? Infect Genet Evol. 2020; 84: 104361. doi: 10.1016/j.meegid.2020.104361.

21. Garcés-Ayala F, Araiza-Rodríguez A, Mendieta-Condado E, RodríguezMaldonado AP, Wong-Arámbula C, Landa-Flores M et al. Full genome sequence of the first SARS-CoV-2 detected in Mexico. Arch Virol. 2020; 165 (9): 2095-2098. doi: 10.1007/s00705-020-04695-3.

22. Taboada B, Vazquez-Perez JA, Muñoz-Medina JE, Ramos-Cervantes P, Escalera-Zamudio M, Boukadida $C$ et al. Genomic analysis of early SARS-CoV-2 variants introduced in Mexico. J Virol. 2020; 94 (18): e01056-20. doi: 10.1128/JVI.01056-20.

23. Zou L, Ruan F, Huang $M$, Liang L, Huang $H$, Hong $Z$ et al. SARSCoV-2 viral load in upper respiratory specimens of infected patients. N Engl J Med. 2020; 382 (12): 1177-1179. doi: 10.1056/ NEJMc2001737.

24. Wölfel R, Corman VM, Guggemos W, Seilmaier M, Zange S, Müller MA et al. Virological assessment of hospitalized patients with COVID-2019. Nature. 2020; 581 (7809): 465-469. doi: 10.1038/ s41586-020-2196-x.

25. Liu Y, Yan LM, Wan L, Xiang TX, Le A, Liu JM et al. Viral dynamics in mild and severe cases of COVID-19. Lancet Infect Dis. 2020; 20 (6): 656-657. doi: 10.1016/S1473-3099(20)30232-2.
26. Vabret N, Britton GJ, Gruber C, Hegde S, Kim J, Kuksin M et al. Immunology of COVID-19: current state of the science. Immunity. 2020; 52 (6): 910-941. doi: 10.1016/j.immuni.2020.05.002.

27. Braun J, Loyal L, Frentsch M, Wendisch D, Georg P, Kurth $\mathrm{F}$ et al. Presence of SARS-CoV-2-reactive T cells in COVID-19 patients and healthy donors. medRxiv. 2020. doi: 10.1101/2020.04.17.20061440.

28. Sekine T, Perez-Potti A, Rivera-Ballesteros O, Stralin K, Gorin JB, Olsson $\mathrm{A}$ et al. Robust $\mathrm{T}$ cell immunity in convalescent individuals with asymptomatic or mild COVID-19. Cell. 2020; 183 (1): 158168.e14. doi: 10.1016/j.cell.2020.08.017.

29. Grifoni A, Sidney J, Zhang Y, Scheuermann RH, Peters B, Sette A. A sequence homology and bioinformatic approach can predict candidate targets for immune responses to SARS-CoV-2. Cell Host Microbe. 2020; 27 (4): 671-680.e2. doi: 10.1016/j. chom.2020.03.002.

30. Chen G, Wu D, Guo W, Cao Y, Huang D, Wang H et al. Clinical and immunological features of severe and moderate coronavirus disease 2019. J Clin Invest. 2020; 130 (5): 2620-2629. doi: 10.1172/JCI137244.

31. Liu J, Li S, Liu J, Liang B, Wang X, Wang $\mathrm{H}$ et al. Longitudinal characteristics of lymphocyte responses and cytokine profiles in the peripheral blood of SARS-CoV-2 infected patients. EBioMedicine. 2020; 55: 102763. doi: 10.1016/j. ebiom.2020.102763. 